\title{
Effect of plastic deformation on stress-induced martensitic transformation of nanocrystalline NiTi alloy
}

\author{
X.B. Shi ${ }^{\mathrm{a}, \mathrm{b}}$, Z.C. $\mathrm{Hu}^{\mathrm{a}}$, X.W. Hu ${ }^{\mathrm{b}}$, J.S. Zhang ${ }^{\mathrm{b}, \mathrm{c}}$, L.S. Cui ${ }^{\mathrm{b}}{ }_{*}$ \\ a School of Materials Science and Engineering, Anhui University of Technology, Maanshan 243002, China \\ b State Key Laboratory of Heavy Oil Processing, China University of Petroleum, Beijing, Changping, 102249, China \\ ${ }^{\mathrm{c}}$ Institute for Advanced Materials, Jiangsu University, Zhenjiang 212013, China
}

\section{A R T I C L E I N F O}

\section{Keywords:}

Nanocrystalline NiTi alloy

Plastic deformation

Lüders-type deformation

Martensitic transformation

Flow stress

\begin{abstract}
A B S T R A C T
A nanocrystalline NiTi alloy with average grain size of $16 \mathrm{~nm}$ was acquired by cold drawing and annealing. The effect of plastic deformation on stress-induced martensitic transformation of the nanocrystalline NiTi alloy was investigated by the tension loading-unloading and heating-cooling cyclic tests. The results reveal that the nanocrystalline NiTi alloy experiences plastic deformation during the tensile loading process. The plastically deformed nanocrystalline NiTi alloy keeps the Lüders-type deformation, which is different from the coarsegrained and ultrafine-grained NiTi alloys.
\end{abstract}

\section{Introduction}

In recent years, nanocrystalline NiTi shape memory alloys have attracted considerable attention of materials scientists [1-7]. The characteristics of thermally-induced martensitic transformations were studied by Waitz et al. and the critical grain size of thermally-induced transformation in nanocrystalline NiTi alloys was analyzed using in-situ TEM [1-3]. From their results, it was proposed that nanocrystalline NiTi alloy has a smallest grain size of about $50 \mathrm{~nm}$ and $15 \mathrm{~nm}$ for B2/ $\mathrm{R} \rightarrow \mathrm{B} 19$ 'and B2 $\rightarrow \mathrm{R}$ transformation, respectively [1-3]. The characteristics of stress-induced martensitic transformations, especially stress hysteresis of nanocrystalline NiTi alloy, were also studied by researchers [4-7]. The stress hysteresis was found to decrease with decreasing grain size in nanocrystalline NiTi alloys, which is closely related to the austenite/martensite phase interfacial energy when transformed incompletely $[4,5]$. Some similar results were acquired by Zhang et al. by molecular dynamics simulation [6]. They found that the stress hysteresis decreases with decreasing sample size in nanoscaled-NiTi, which related to the surface energy of the samples [6]. Different from these results, the stress hysteresis of nanocrystalline NiTi alloy, which experiences complete martensitic transformation, was found to increase with decreasing grain size [7]. All the results reviewed above support that nanocrystalline NiTi alloys experience different characteristics of both thermally-induced and stress-induced martensitic transformations compared to their large-grain counterparts.

The effect of plastic deformation on martensitic transformations of coarser-grained and ultrafine-grained NiTi alloys has been studied by many researchers [8-14]. The generation and storage of dislocations can significantly affect the transformation characteristics of the coarsergrained and ultrafine-grained NiTi alloys [8-14].In the recent years, the research shows that, the deformation mechanisms of nanocrystalline materials are radically different from conventional coarser-grained materials, as plasticity at the nanoscale maybe mediated mostly by grain-boundary deformation processes [15-22]. In nanocrystalline metals or alloys, full dislocations are difficult to generate or slide, the dominant plastic mechanism tends to the slide of partial dislocations and the deformation of grain boundary [15-26]. Besides, the extremely small grain size makes the nanocrystalline materials difficult to store dislocations [16-26].It is meaningful that whetherthis effect of dislocations on the martensitic transformation characteristics is compatible with the nanocrystalline NiTi alloys. In this paper, effect of plastic deformation on martensitic transformation of nanocrystalline NiTi alloy was studied by tensile testing, differential scanning calorimeter (DSC) experiment and transmission electron microscopy(TEM) observation. The dislocation movement and grain boundary deformation were discussed, which may affect the shape of stress-strain curves of the nanocrystalline NiTi alloys [8-14,27].

\section{Experimental Procedures}

A near-equiatomic NiTi alloy wire of $1.0 \mathrm{~mm}$ in diameter with a nominal composition of $\mathrm{Ni}-49.8 \mathrm{at} \% \mathrm{Ti}$ was acquired from the General Research Institute for Non-Ferrous Metals, China. The wire was annealed at $750{ }^{\circ} \mathrm{C}$ for $90 \mathrm{~s}$ followed by air cooling and then cold

\footnotetext{
* Corresponding author.

E-mail address: lishancui63@126.com (L.S. Cui).
} 

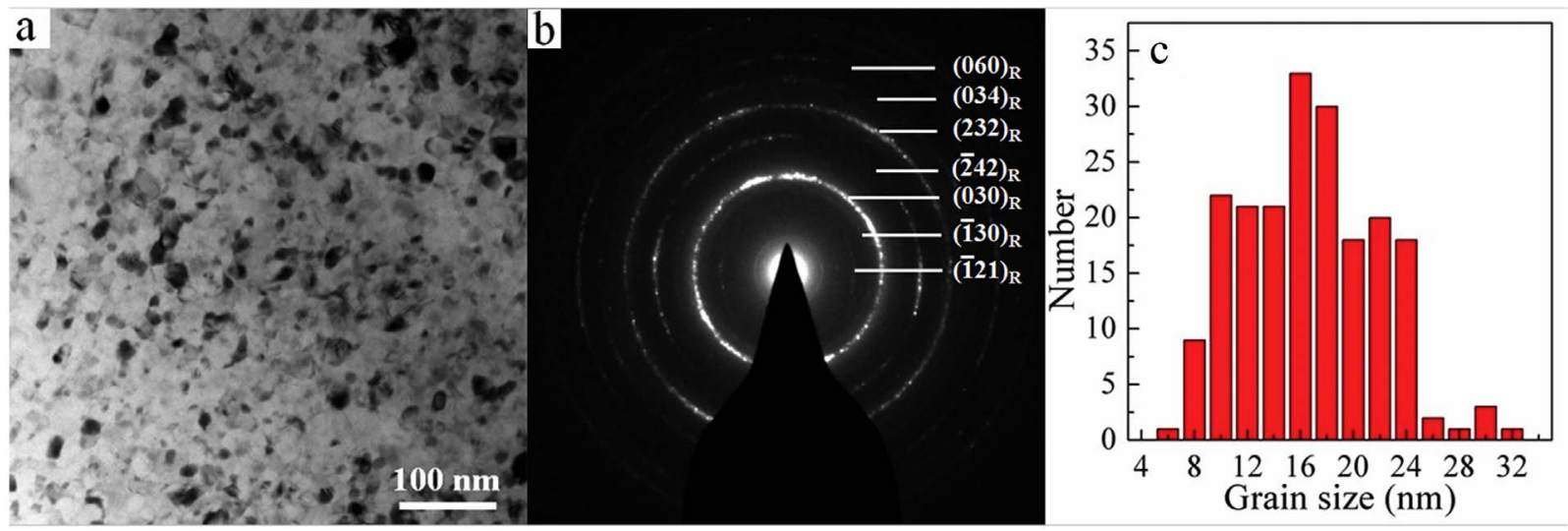

Fig. 1. The microstructure analysis of the $350{ }^{\circ} \mathrm{C}$ annealed NiTi alloy, (a) TEM Bright-field image; (b) SAED pattern and (c) grain size distribution.

drawn into diameter of $0.55 \mathrm{~mm}$. The samples cut from the cold-drawn wire were annealed in air at $350{ }^{\circ} \mathrm{C}$ for $0.6 \mathrm{ks}$. The tensile tests were carried out using a WDT II-20 type tensile machine using a tensile speed of about $2.5 \% / \mathrm{min}$ at room temperature. The flow stress for martensitic transformation was acquired by calculating the average value of the upper platform stress in the stress-strain curves. The DSC experiments of the samples before and after tensile deformation were carried out using DSC Q20 (TA Instrument) thermal analyzer in a nitrogen atmosphere. Samples (before and after tensile deformation) used for TEM observation were mechanically polished and then ion milled using a Gatan PIPS. The TEM observation was carried out using a FEI Tecnai F20 (operating at $200 \mathrm{kV}$ ) equipped with a Gatan slow scan CCD camera.

\section{Results and Discussion}

Fig. 1 shows the grain size analysis of the $350{ }^{\circ} \mathrm{C}$ annealed sample using TEM method. Fig. 1(a) and (b) show the TEM bright field image and the corresponding selected area electron diffraction (SAED) pattern. From the bright field image, it is seen that small grains distributed uniformly in the annealed sample. From the indexes in Fig. 3(b), it can be seen that the diffraction rings all belong to $\mathrm{R}$ phase. Fig. 1(c) is the grain size distribution by measuring 200 grains of the annealed sample. It is shown that most grains are smaller than $25 \mathrm{~nm}$ and the average grain size is about $16 \mathrm{~nm}$.

Fig. 2 is the results of tensile test and DSC analysis of the nanocrystalline NiTi alloy. Fig. 2(a) shows the 16\% tensile stress-strain curve and the DSC analysis of the samples before and after deformation. From the tensile stress-strain curve, it can be seen that the sample experiences two stages yielding upon loading. It is proposed that the first yielding corresponds to an R-phase orientation and the second yielding corresponds to a martensitic transformation. The unloading curve is near-linear and the residual strain is about $11.3 \%$ after unloading, which indicates that the reverse martensitic transformation doesn't proceed. The DSC analysis in the inset of Fig. 2(a) shows transformation characteristics of the samples before and after deformation. It can be seen that the as-annealed sample experiences a reversible phase transformation upon heating-cooling cycle at the temperature range of $0-100^{\circ} \mathrm{C}$. The temperature hysteresis of the transformation is about $6{ }^{\circ} \mathrm{C}$, which indicates that the sample experiences a $\mathrm{B} 2 \leftrightarrow \mathrm{R}$ transformation. The red curve shows the transformation behavior of the deformed sample upon heating. It can be seen that the red curve exhibits two endothermic peaks. Similar to the result reported recently [28], we can propose that the deformed sample experiences a two-stage B19' $\rightarrow$ B2 transformation upon heating.

Fig. 2(b) shows the results of cyclic tests of the nanocrystalline NiTi alloy. Here, the sample experiences 6 cycles. In each cycle, the sample experiences a tensile loading-unloading test followed by a heating- cooling process. The heating temperature is about $120{ }^{\circ} \mathrm{C}$, which insure the martensitic phase inverse transformed completely. Then, the sample was cooled to room temperature in air, waiting for the next cycle. From the tensile loading-unloading curves, it is seen that the samples do not experience the early yielding process that corresponds to the R-phase orientation at the cycles $2-6$. We propose that internal stress induces Rphase reorientation after the cycle 1 , which results in the absence of the early yield in the following cycles. The black curve in Fig. 2(c) shows the relationship between flow stress for martensitic transformation and the cycle number. The flow stress decreases with increasing of the cycle times and the flow stress of the cycle 5 ( $287 \mathrm{MPa}$ ) is much close to that of the cycle $6(286 \mathrm{MPa})$. The decrease of flow stress after the cycles indicates that the nanocrystalline NiTi alloy experiences plastic deformation during loading [8-10,27].The inset of Fig. 2(c) shows the schematic of dissipated energy for plastic deformation of the cycle $n$ $\left(\mathrm{E}_{\mathrm{p}}{ }^{n}\right)$. The gray area indicates the total dissipated energy $\mathrm{E}^{n-1}$ of the cycle $n-1$. The blue area indicates the dissipated energy difference $\left(\mathrm{E}^{n}-\mathrm{E}^{n-1}\right)$ between the cycle $n$ and $n-1$. We propose that the dissipated energy difference $E^{n}-E^{n-1}$ indicates the dissipated energy for irreversible deformation, which corresponds to the dissipated energy for plastic deformation $\left(\mathrm{E}_{\mathrm{p}}{ }^{n}\right)$. The red curve in Fig. 2(c) is the evolution of $\mathrm{E}_{\mathrm{p}}{ }^{n}$ during loading with the cycle number. From the red curve, it can be seen that $\mathrm{E}_{\mathrm{p}}{ }^{1}$ is about $46.6 \mathrm{MJ} / \mathrm{m}^{3}$, which indicates that significantly plastic deformation occurs during the 1st loading cycle. The dissipated energy for plastic deformation $E_{p}$ decreases with the increasing of the cycle number, which indicates that plastic deformation decreases with the increasing cycle number. The dissipated energy $\mathrm{E}_{\mathrm{p}}{ }^{5}$ is about $0.4 \mathrm{MJ} / \mathrm{m}^{3}$, which indicates that few plastic deformation occurs during the cycle 5 .

All the results described above support that nanocrystalline NiTi alloy experiences plastic deformation during the tensile loading process, and after 4 cycles, few plastic deformation occurs and the tensile curve keep stable. As the results reported before [8-10,27], when tension with a large strain (larger than 10\%), the plastic processes change the deformation modes from localized to homogeneous at the 2nd or 3rd cycle in the ultra-fine grained and coarse-grained NiTi alloys. It was proposed that the accumulation of dislocation defects in the NiTi alloys induces the homogeneous deformation mode [8-10]. Besides, the effect of redistribution of the internal stress, which induces the accumulation of defects and residual martensite was also proposed [27]. The TEM results reported by Delville et al. [8] show that dislocations generated and accumulated in the deformed ultra-fine grained and coarse-grained NiTi alloys. Some in-situ electron backscatter diffraction (EBSD) analysis reported by Mao et al. [29,30] also suggest that plastic deformation inhibits the Lüders-type martensitic transformation in coarse-grained NiTi alloys. Their results show that martensitic traces, a typical character of plastic deformation, exist inside coarse grains of NiTi alloys after unloading [29,30]. High density 

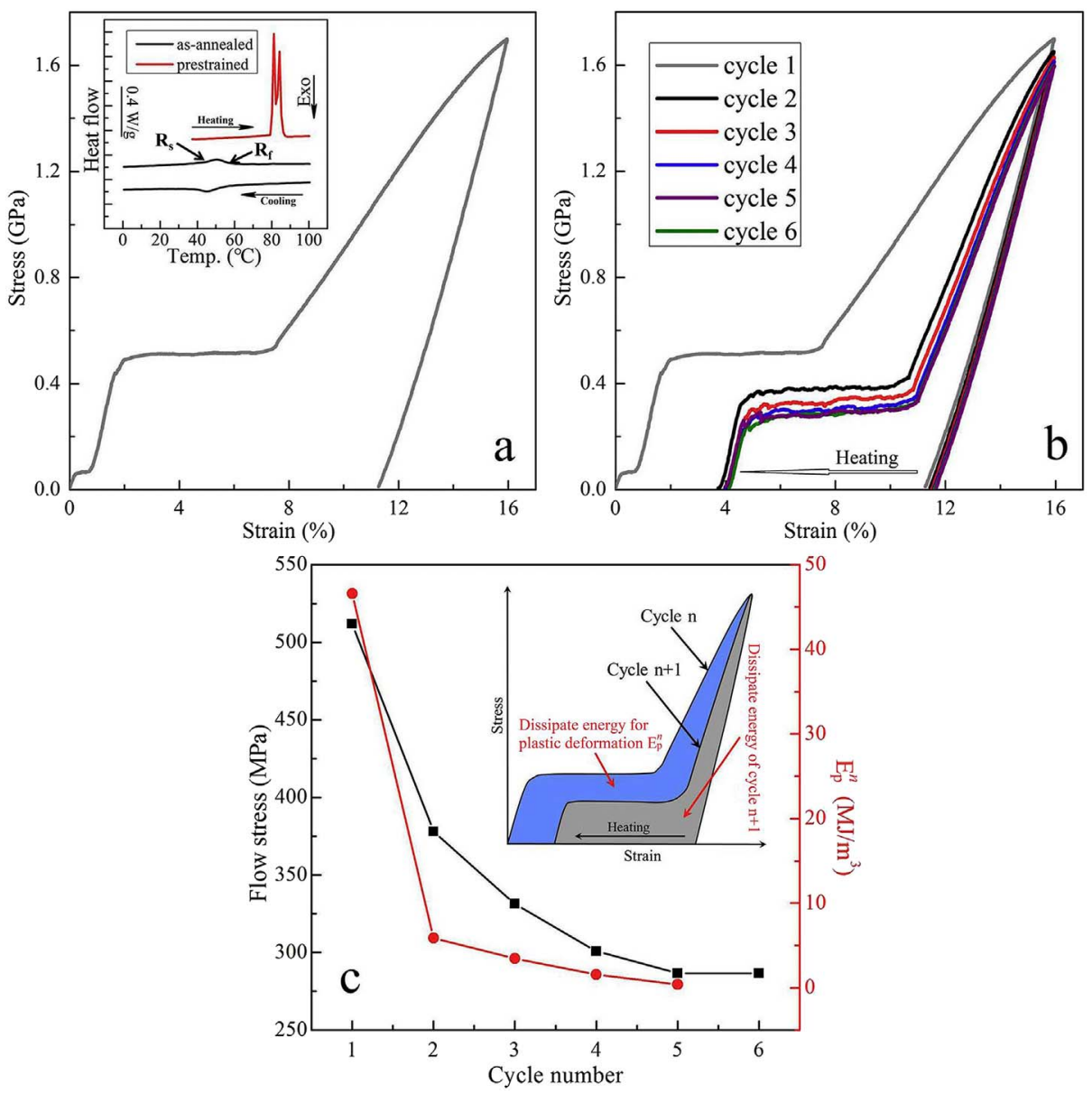

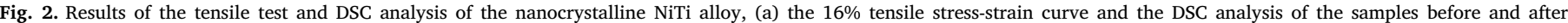

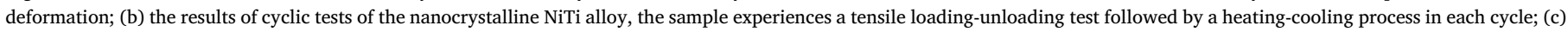

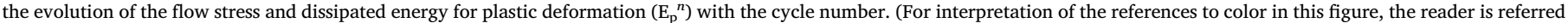
to the web version of this article.)

defects in the martensitic traces will inhibit the cooperative-collective effect among grains and the Lüders-type martensitic transformation $[29,30]$. Here, different from the NiTi alloys with larger grains [8-10], we can see that the stress-strain curves of the nanocrystalline NiTi alloy exhibit stress plateaus during the loading processes at all of the cycles, which indicates a Lüders-type deformation. Fig. 3 shows the microstructure analysis of the nanocrystalline NiTi alloy after one cyclic test with $16 \%$ strain. Fig. 3(a) is the TEM bright field image of the sample. By contrast to the bright field image in Fig. 1(a), we can see that the samples before and after the cyclic test have the same microstructure. The corresponding SAED pattern in Fig. 3(b) shows the R-phase diffraction rings, which are same to that of the as-annealed sample shown in Fig. 1(a). No martensitic diffraction ring was found in the SAED pattern, which indicates that there is no residual martensite existed in the cycled sample. In order to investigate the microstructure of the cycled sample, we have observed several grains by high resolution TEM (HRTEM) method. Fig. 3(c) and (d) show the high resolution TEM (HRTEM) images of two grains of the cycled sample. From the HRTEM images we can see that there is no dislocation and no residual martensite phase in the nanograins after the tension cycle, which is different from the microstructures of the ultrafine-grained and coarse-grained NiTi alloys after deformation $[8,27]$. This result suggests that, in nanocrystalline NiTi alloy, dislocations do not accumulate in the nanograins during tension loading-unloading process, which lead the cycled nanocrystalline NiTi alloy experiences a Lüders-type deformation during loading. This explanation is consistent with the theory reported recently, which proposes that dislocations nucleate at one side of the grain, traverse the grain, and then disappear into the grain boundaries on the opposing side, such that no dislocation is left in nanocrystalline metals or alloys [16-26].

However, it is hard to explain why the flow stress decreases with the increasing cycle number, when there is no dislocation and no residual martensite phase left in the nanograins. As was reported recently $[31,32]$, a similar result was observed in a NiTi alloy with mixed grain sizes. Three deformation mechanisms on transforming related to the generation of dislocations were proposed [31,32]: (i) Facilitating the formation and growth of the martensite. (ii) Providing the mechanisms for grain shape accommodation. (iii) Providing the plastic work for the accommodation of the non-transforming precipitates in the martensite matrix. Here, in the NiTi alloys, precipitate was not found. We propose that the traverse slide of dislocations changes the shape and orientation of the nanograins, which will facilitate the formation and growth of the martensite and provide the mechanisms for grain shape accommodation on martensitic transforming, and then reduces the transformation stress. Besides, in the nanocrystalline metals and alloys, it is proposed that, the plastic deformation of grain boundary (including grain boundary sliding, grain boundary diffusion and grain rotation) occurs especially in the grains smaller than $50 \mathrm{~nm}$ [16-26]. We deduce that the plastic deformation of grain boundary may occur in nanocrystalline NiTi alloy during tension to a high strain (16\%). When the inverse transformation occurs during the heating process, the plastically deformed grain boundaries impede the axial shrink of the nanograins. The nanograins bear tensile internal stress after the heating process. Therefore, the cycled sample needs a lower flow stress to induce the martensitic transformation. 


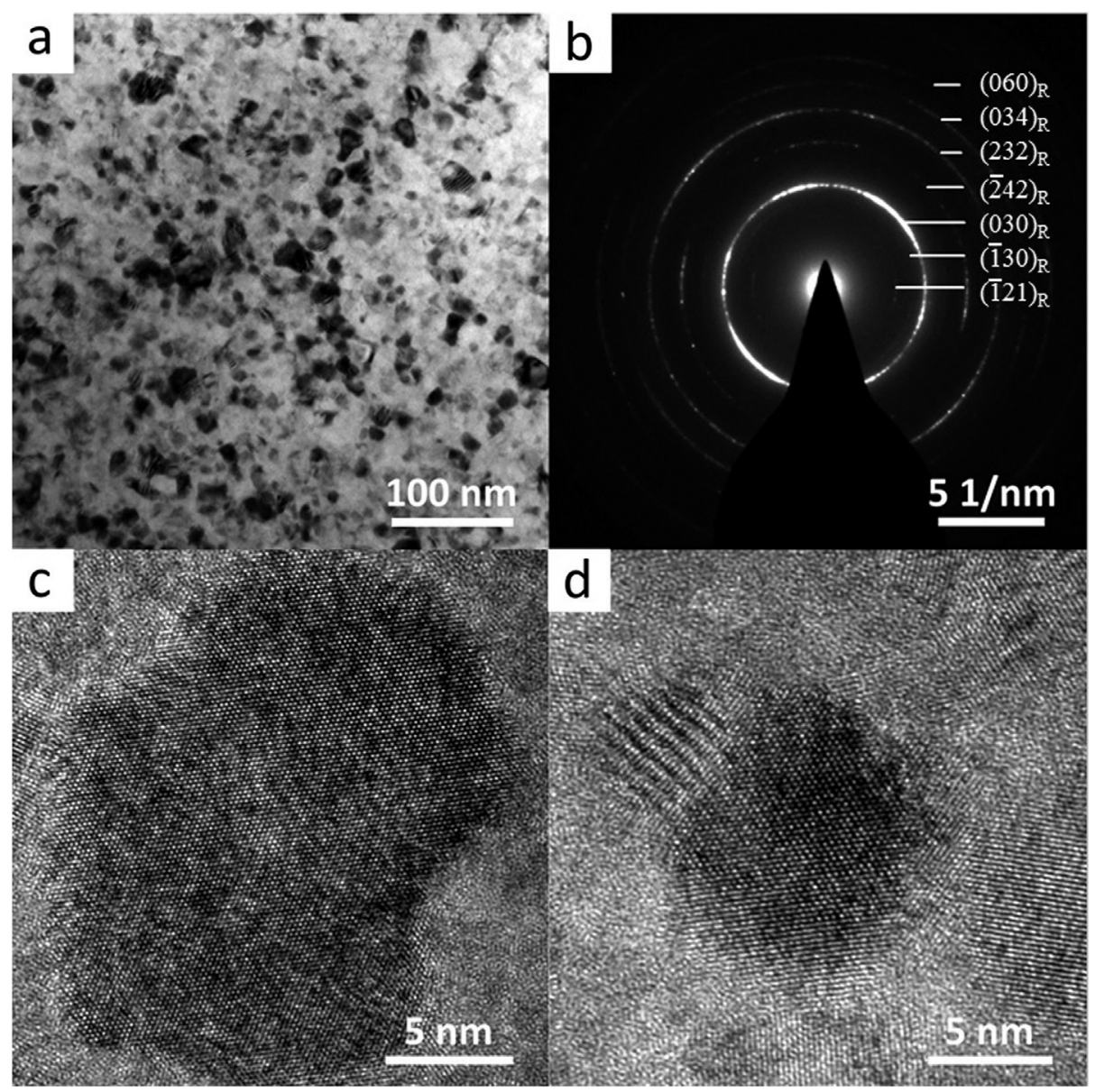

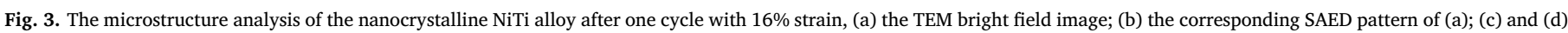
the HRTEM images of two nanograins.

\section{Conclusions}

Effect of plastic deformation on the stress-induced martensitic transformation of nanocrystalline NiTi alloy with an average grain size of $16 \mathrm{~nm}$ was investigated by the tension loading-unloading and heating-cooling cyclic tests. The results show that the flow stress and dissipated energy decrease with the increasing cycle number, which suggest that the sample experiences plastic deformation. The plastically deformed sample keeps the Lüders-type deformation, which is different from the coarse-grained and ultrafine-grained NiTi alloys. The microstructure analysis supports that dislocations do not accumulate in the nanograins during tension loading-unloading process. The traverse slide of dislocations across the nanograins was proposed in the plastic deformation process, which in turn will facilitate the formation and growth of the martensite and provide the mechanisms for grain shape accommodation on martensitic transforming, and then reduces the transformation stress. Besides, the plastic deformation of grain boundaries may occur during tension to a high strain (16\%), which will reduce the transformation stress.

\section{Acknowledgment}

This work was supported by the National Science Foundation of China (NSFC) (Grant No. 51601001 and 51601069).

\section{References}

[1] T. Waitz, T. Antretter, F.D. Fischer, N.K. Simha, H.P. Karnthaler, J. Mech, Phys. Solids 55 (2007) 419-444.

[2] T. Waitz, H.P. Karnthaler, Acta Mater. 52 (2004) 5461-5469.
[3] T. Waitz, V. Kazykhanov, H.P. Karnthaler, Acta Mater. 52 (2004) 137-147.

[4] Q.P. Sun, Y.J. He, Amultiscale continuum model of the grain-size dependence of the stress hysteresis in shape memory alloy polycrystals, Int. J. Solids Struct. 45 (2008) 3868-3896.

[5] A. Ahadi, Q.P. Sun, Stress hysteresis and temperature dependence of phase transition stress in nanostructured NiTi-effects of grain size, Appl. Phys. Lett. 103 (2013) 021902.

[6] Z. Zhang, X. Ding, J. Sun, T. Suzuki, T. Lookman, K. Otsuka, X. Ren, Nonhysteretic superelasticity of shape memory alloys at the nanoscale, Phys. Rev. Lett. 111 (2013) 145701.

[7] X.B. Shi, F.M. Guo, J.S. Zhang, H.L. Ding, L.S. Cui, Grain size effect on stress hysteresis of nanocrystalline NiTi alloys, J. Alloys Compd. 688 (2016) 62-68.

[8] R. Delville, B. Malard, J. Pilch, P. Sittner, D. Schryvers, Transmission electron microscopy investigation of dislocation slip during superelastic cycling of $\mathrm{Ni}-\mathrm{Ti}$ wires, Int. J. Plast. 27 (2011) 282-297.

[9] G. Eggeler, E. Hornbogen, A. Yawny, A. Heckmann, M. Wagner, Structural and functional fatigue of NiTi shape memory alloys, Mater. Sci. Eng. A 378 (2004) 24-33.

[10] K. Gall, H.J. Maier, Cyclic deformation mechanisms in precipitated NiTi shape memory alloys, Acta Mater. 50 (2002) 4643-4657.

[11] P. Sittner, Y. Liu, V. Novak, On the origin of Lüders-like deformation of NiTi shape memory alloys, J. Mech. Phys. Solids 53 (2005) 1719-1746.

[12] G. Tan, Y. Liu, P. Sittner, M. Saunders, Lüders-like deformation associated with stress-induced martensitic transformation in NiTi, Scr. Mater. 50 (2004) 193-198.

[13] B. Kockar, I. Karaman, J.I. Kim, Y.I. Chumlyakov, J. Sharp, C.J. (Mike) Yu, Thermomechanical cyclic response of an ultrafine-grained NiTi shape memory alloy, Acta Mater. 56 (2008) 3630-3646.

[14] L.C. Brinson, I. Schmidt, R. Lammering, Stress-induced transformation behavior of a polycrystalline NiTi shape memory alloy: micro and macro mechanical investigations via in situ optical microscopy, J. Mech. Phys. Solids 52 (2004) 1549-1571.

[15] H. Li, H. Choo, Y. Ren, T.A. Saleh, U. Lienert, P.K. Liaw, F. Ebrahimi, Strain dependent deformation behavior in nanocrystalline metals, Phys. Rev. Lett. 101 (2008) 015502

[16] M. Dao, L. Lu, R.J. Asaro, D. Hosson, E. Ma, Toward a quantitative understanding of mechanical behavior of nanocrystalline metals, Acta Mater. 55 (2007) 41-65.

[17] K.S. Kumar, H. Van Swygenhoven, S. Suresh, Mechanical behavior of nanocrystalline metals and alloys, Acta Mater. 51 (2003) 5743-5774.

[18] K.S. Kumar, S. Suresh, M.F. Chisholm, J.A. Horton, P. Wang, Deformation of electro deposited nanocrystalline nickel, Acta Mater. 51 (2003) 387-405. 
[19] Z. Budrovic, H. Van Swygenhoven, P.M. Derlet, S. Van Petegem, B. Schmitt, Plastic deformation with reversible peak broadening in nanocrystalline nickel, Science 304 (2004) 273-276.

[20] L. Wang, X. Han, P. Liu, Y. Yue, Z. Zhang, E. Ma, In situ observation of dislocation behavior in nanometer grains, Phys. Rev. Lett. 105 (2010) 135501.

[21] L. Wang, J. Teng, P. Liu, A. Hirata, E. Ma, Z. Zhang, M. Chen, X. Han, Grain rotation mediated by grain boundary dislocations in nanocrystalline platinum, Nat. Commun. 5 (2014) 4402.

[22] P. Liu, S. Mao, L. Wang, X. Hana, Z. Zhang, Direct dynamic atomic mechanisms of strain-induced grain rotation in nanocrystalline, textured, columnar-structured thin gold films, Scr. Mater. 64 (2011) 343-346.

[23] K. Zheng, X. Han, L. Wang, Y. Zhang, Y. Yue, Y. Qin, X. Zhang, Z. Zhang, Atomic mechanisms governing the elastic limit and the incipient plasticity of bending $\mathrm{Si}$ nanowires, Nano Lett. 9 (2009) 2471-2476.

[24] L. Wang, P. Liu, P. Guan, M. Yang, J. Sun, Y. Cheng, A. Hirata, Z. Zhang, E. Ma, M. Chen, X. Han, In situ atomic-scale observation of continuous and reversible lattice deformation beyond the elastic limit, Nat. Commun. 4 (2013) 2413.

[25] Y. Yue, P. Liu, Z. Zhang, X. Han, E. Ma, Approaching the theoretical elastic strain limit in copper nanowires, Nano Lett. 11 (2011) 3151-3155.

[26] Z. Shan, R. Mishra, S. Asif, O. Warren, A. Minor, Mechanical annealing and source- limited deformation in submicrometre-diameter Ni crystals, Nat. Mater. 7 (2007) $115-119$.

[27] P. Sedmák, P. Šittner, J. Pilch, C. Curfs, Instability of cyclic superelastic deformation of NiTi investigated by synchrotron X-ray diffraction, Acta Mater. 94 (2015) 257-270.

[28] X.B. Shi, M.Y. Yu, F.M. Guo, Z.Y. Liu, D.Q. Jiang, X.D. Han, L.S. Cui, Effect of deformation on the stability of stress-induced martensite in nanocrystalline NiTi shape memory alloy, Mater. Lett. 131 (2014) 233-235.

[29] S. Mao, J. Luo, Z. Zhang, M. Wu, Y. Liu, X. Han, EBSD studies of the stress-induced B2-B19' martensitic transformation in NiTi tubes under uniaxial tension and compression, Acta Mater. 58 (2010) 3357-3366.

[30] S. Mao, X. Han, Z. Zhang, The nano - and mesoscopic cooperative collective mechanisms of inhomogenous elastic-plastic transitions in polycrystalline TiNi shape memory alloys, J. Appl. Phys. 101 (2007) 103522.

[31] A. Yawny, J. Olbricht, M. Sade, G. Eggeler, Pseudoelastic cycling and ageing effects at ambient temperature in nanocrystalline Ni-rich NiTi wire, Mater. Sci. Eng. A 481-482 (2008) 86-90.

[32] A.M. Condó, F.C. Lovey, J. Olbricht, Ch. Somsen, A. Yawny, Microstructural aspects related to pseudoelastic cycling in ultra fine grained Ni-Ti, Mater. Sci. Eng. A 481-482 (2008) 138-141. 